Research Paper

\title{
Bromodomain inhibitor OTX015 (MK-8628) combined with targeted agents shows strong in vivo antitumor activity in Iymphoma
}

\author{
Eugenio Gaudio ${ }^{1}$, Chiara Tarantelli ${ }^{1}$, Maurilio Ponzoni ${ }^{2}$, Elodie Odore ${ }^{3}$, Keyvan \\ Rezai $^{3}$, Elena Bernasconi ${ }^{1}$, Luciano Cascione ${ }^{1,4}{ }^{,}$Andrea Rinaldi ${ }^{1}$, Anastasios Stathis ${ }^{4}$, \\ Eugenia Riveiro5, Esteban Cvitkovic ${ }^{5}$, Emanuele Zucca ${ }^{4}$, Francesco Bertoni ${ }^{1,4}$ \\ ${ }^{1}$ Lymphoma and Genomics Research Program, Institute of Oncology Research (IOR), Bellinzona, Switzerland \\ ${ }^{2}$ San Raffaele Scientific Institute, Milan, Italy \\ ${ }^{3}$ Institut Curie, Hôpital René Huguenin, Saint-Cloud, France \\ ${ }^{4}$ Oncology Institute of Southern Switzerland (IOSI), Bellinzona, Switzerland \\ ${ }^{5}$ Oncology Therapeutic Development, Clichy, France \\ Correspondence to: Francesco Bertoni, email: frbertoni@mac.com \\ Keywords: BET inhibitor, ibrutinib, rituximab, vorinostat, everolimus \\ Received: April 28, $2016 \quad$ Accepted: July 07, $2016 \quad$ Published: August 1, 2016
}

\section{ABSTRACT}

The bromodomain inhibitor OTX015 (MK-8628) has shown anti-lymphoma activity as a single agent in both the preclinical and clinical settings, as well as in vitro synergism with several anticancer agents. Here, we report in vivo data for OTX015 in combination with the histone deacetylase inhibitor vorinostat, the Bruton's tyrosine kinase inhibitor ibrutinib, the anti-CD20 monoclonal antibody rituximab, and the mTOR inhibitor everolimus in a diffuse large B cell lymphoma model. The antitumor effect of OTX015-containing combinations in SU-DHL-2 xenografts in mice was much stronger than the activity of the corresponding single agents with almost complete tumor eradication for all four combinations. Pharmacokinetic analyses showed similar OTX015 levels in plasma and tumor samples of approximately 1.5 $\mu \mathrm{M}$, which is equivalent to the concentration showing strong in vitro activity. For all four combinations, mean terminal levels of the bromodomain inhibitor differed from those in mice exposed to single agent OTX015, indicating a need for thorough pharmacokinetic investigations in phase I combination studies. In conclusion, our results provide a strong rationale to explore OTX015-containing combinations in the clinical lymphoma setting.

\section{INTRODUCTION}

Diffuse large B cell lymphoma (DLBCL) is the most common lymphoma in Western countries and, despite improvements obtained with chemoimmunotherapy, up to half of these patients cannot be cured [1,2]. Currently, two main DLBCL subtypes are recognized based on their phenotypic homology with their putative cell of origin, the germinal center B-cell type and the activated B-cell like $(\mathrm{ABC})$ type [2-5]. ABC-DLBCL is less responsive to standard regimens and is characterized by activation of B-cell receptor signaling and the nuclear factor $\mathrm{kB}$ pathway [2-5], providing therapeutic targets that are currently being explored in the clinic with compounds such as the Bruton's tyrosine kinase (BTK) inhibitor ibrutinib [6].

Analogous to the vast majority of human tumors and independent of their cell of origin, DLBCL cells also bear recurrent somatic mutations in genes coding for proteins involved in chromatin structure and remodeling that cause profound changes at the epigenetic level [3, 7]. Of clinical relevance, epigenetic changes can be at least partially reversed and epigenetic drugs can increase sensitivity to other anticancer agents [7-10]. Over the last few years, 
inhibitors of the bromodomain and extraterminal (BET) protein family have become the focus of extensive research as a novel class of epigenetic drugs [11]. BET proteins are key epigenetic regulators of gene transcription and their inhibition has resulted in antitumor activity in different tumor models, including lymphomas [11-20]. OTX015 (MK-8628) is a thienotriazolodiazepine compound that potently inhibits the BET proteins BRD2, BRD3 and BRD4 [21]. The compound competitively occupies the acetyl-binding pockets of BET bromodomains, leading to release of the BET protein from the chromatin [21]. Importantly, in normal and cancer cells, more than half of all BRD4 proteins are bound to a small number of enhancers (super-enhancers) that control the expression of genes fundamental to the control and establishment of individual cell identities, such as PAX5, BCL6, CD79A, CD79B, FOXO1 in B-cells or PRDM1, IRF4 and MUM1 in plasma cells [22-24].

OTX015 has in vitro and in vivo antitumor activity as a single agent in different lymphoma models, including ABC-DLBCL [14]. Clinical responses including complete remissions with single agent OTX015 have been recently reported in patients with relapsed or refractory lymphoma or acute leukemia enrolled in phase I studies, in the absence of major toxicities $[25,26]$. Although the mechanism of action of BET inhibitors is likely pleiotropic, down-regulation of genes involved in B cell identity and germinal center formation, and, especially in the ABC-DLBCL setting in which such effects can lead to apoptosis, inhibition of the B-cell receptor and nuclear factor $\mathrm{kB}$ signaling pathways play an important role [12-14]. Since OTX015 presented in vitro synergism when combined with different agents in lymphoma models [14], we evaluated the in vivo activity of OTX015-containing combinations in an ABC-DLCBL xenograft model.

\section{RESULTS AND DISCUSSION}

Based on the in vitro synergism observed for combinations of OTX015 with other compounds [14], we evaluated the activity of combinations of this bromodomain inhibitor in an in vivo model of ABC-DLBCL. Mice bearing xenografts of the ABCDLBCL cell line SU-DHL-2 were treated with control or OTX015, BTK inhibitor ibrutinib, the mechanistic target of rapamycin (mTOR) inhibitor everolimus, the histone deacetylase inhibitor vorinostat, or the antiCD20 monoclonal antibody rituximab as single agents or in OTX015-containing combinations. None of the mice showed any body weight loss during the treatment period. When given as single agents, OTX015 and all four other drugs caused tumor growth delay (Figure 1A). When given in combination, the antitumor activity was significantly greater, with an almost complete and immediate tumor eradication in mice receiving the OTX015-containing combinations, maintained throughout treatment $(\mathrm{P}<0.001)$ (Figure 1A). The degree of necrosis was also evaluated in three tumors per group. Tumors from mice treated with rituximab $(\mathrm{P}=0.0463)$, everolimus $(\mathrm{P}=0.0463)$ or ibrutinib $(\mathrm{P}=0.0431)$ as single agents, or with OTX015 combinations plus everolimus $(\mathrm{P}=0.0463)$, plus ibrutinib $(\mathrm{P}=0.0431)$, and plus vorinostat $(\mathrm{P}=0.0463)$ presented a higher percentage of necrotic cells than control mice (Figure 1B, 1C). Higher necrosis was observed in tumors from mice treated with the OTX015 and vorinostat combination compared to the single agent vorinostat group $(\mathrm{P}=0.0109)$. Together with our previous in vitro findings with OTX015 as a single agent and in combination [14], the OTX015 antitumor activity reported as single agent in the phase I hematologic study [25], and similar positive results of other combination regimens based on BET inhibitors [13, 16-18], these novel in vivo data confirm the combinability of OTX015 with classic cytotoxic and targeted therapies in lymphoma and provide supporting rationale for future clinical development strategies in lymphoma. Due to the direct effect of OTX015 and other BET inhibitors on MYC expression, independently of the presence of chromosomal translocations [14], also high-risk populations such the double-hit or doubleexpressor lymphomas [4] could be targeted.

Pharmacokinetics analyses showed similar OTX015 levels in plasma and tumor samples $4 \mathrm{~h}$ after the last treatment when administrated as a single agent, with values of $\sim 750 \mathrm{ng} / \mathrm{ml}$ in plasma, which is equivalent to the $1.5 \mu \mathrm{M}$ concentration that has strong in vitro activity [14], and $\sim 750 \mathrm{ng} / \mathrm{g}$ of tissue for tumor samples (Figure 2A-2B). Terminal levels of the bromodomain inhibitor in all experimental groups treated with OTX015 in combination, differed from the group exposed to OTX015 as single agent. Co-treatment with ibrutinib or everolimus induced an increase in OTX015 concentrations both in plasma (Figure 2A) and tumor samples (Figure 2B). On the other hand, treatment with rituximab decreased OTX015 accumulation in the tumor tissue and OTX015 was not detected in plasma samples in mice concomitantly receiving vorinostat. These results are based on a limited number of mice but suggest that extended pharmacokinetic/ pharmacodynamic studies should be mandatory in phase I combination studies to explore the behavior of OTX015 when administered with other agents.

In conclusion, OTX015 showed strong in vivo activity in a murine xenograft model of ABC-DLBCL when combined with ibrutinib, everolimus, rituximab, or vorinostat. Our results provide the rationale to explore OTX015-containing combinations in the clinical setting. 


\section{MATERIALS AND METHODS}

NOD-Scid (NOD.CB17-Prkdcscid/NCrHsd) mice (five weeks of age, approximately $20 \mathrm{~g}$ body weight; Harlan Laboratory, S. Pietro al Natisone, Udine, Italy) were subcutaneously engrafted with $15 \times 10^{6}$ cells of the human ABC-DLBCL cell line SU-DHL-2, and randomly divided into 10 groups of six mice each. Treatment with OTX015 $(50 \mathrm{mg} / \mathrm{kg}$ once daily, oral, $\mathrm{qdx} 7 / \mathrm{w} \times 5 \mathrm{w}$; Oncoethix
GmbH, a wholly owned subsidiary of Merck Sharp \& Dohme Corp, Lucerne, Switzerland; formerly Oncoethix SA) was initiated three days after the engraftment, while treatment with ibrutinib $(5 \mathrm{mg} / \mathrm{kg} \mathrm{PO} ; \mathrm{qdx} 2 / \mathrm{w} \times 5 \mathrm{w})$, everolimus ( $1 \mathrm{mg} / \mathrm{kg}$ PO; qdx $2 / \mathrm{w} \times 5 \mathrm{w})$, vorinostat $(15 \mathrm{mg} /$ kg PO; qdx2/w x $5 w$; Selleckchem, Houston, TX, USA) or rituximab ( $3 \mathrm{mg} / \mathrm{kg} \mathrm{IV;} \mathrm{qdx1/w} \mathrm{x5w;} \mathrm{Roche,} \mathrm{Basel,}$ Switzerland) was initiated when mice developed palpable tumors $\left(100 \mathrm{~mm}^{3}\right)$. Tumor size was measured twice weekly
A
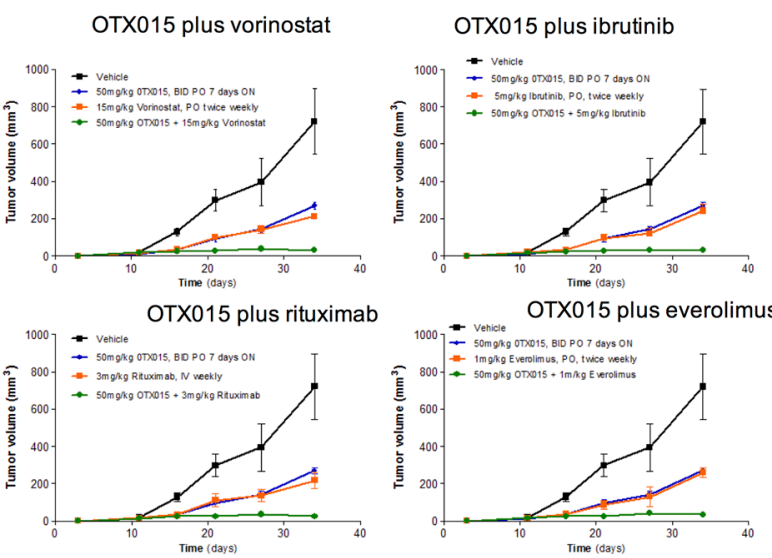

B

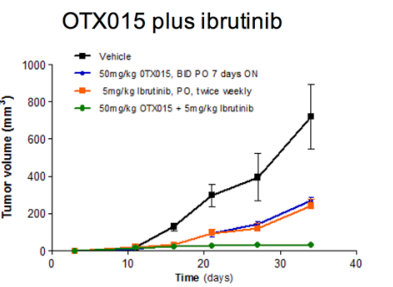

OTX015 plus everolimus

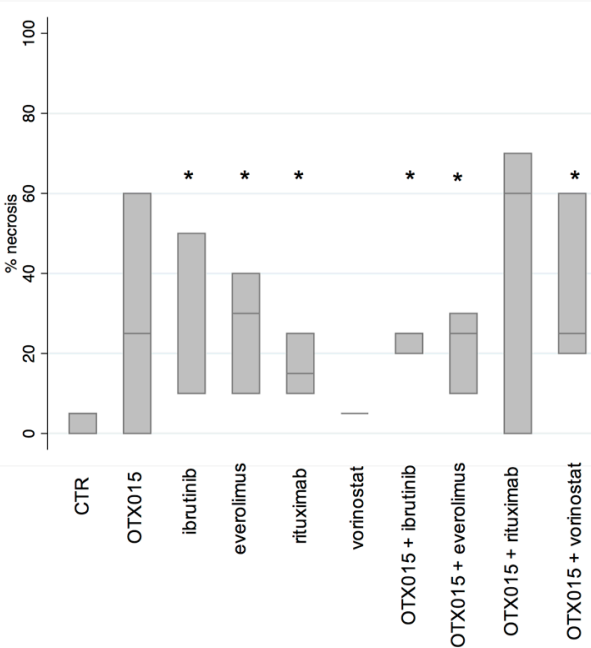

C
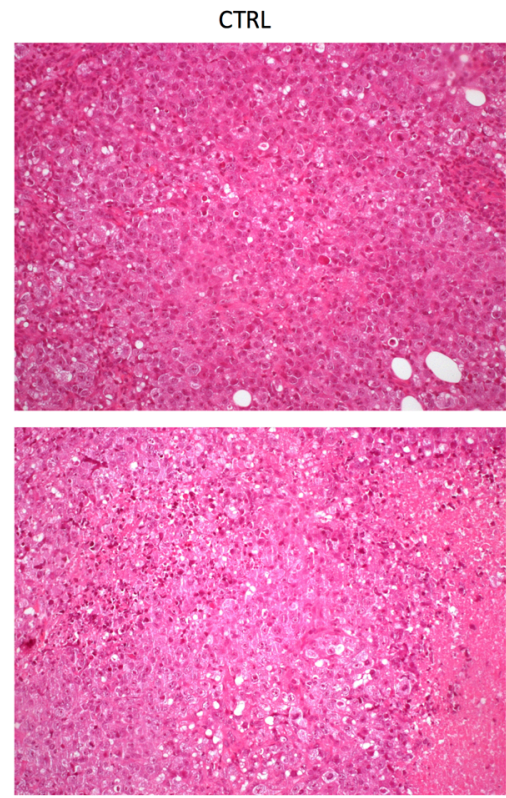

Rituximab_3mg/Kg IV
OTX015_50mg/Kg PO
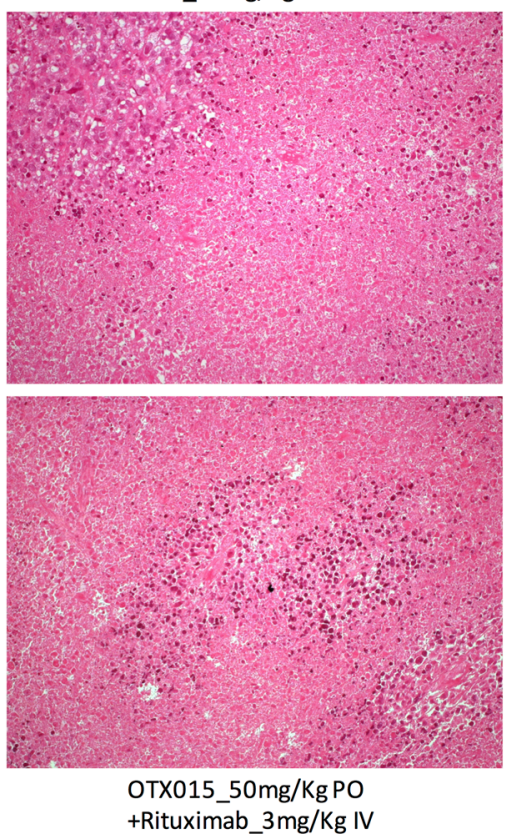

Figure 1: In vivo treatment of ABC-DLBCL SU-DHL-2 xenografts with OTX015 as a single agent and in combination with other targeted drugs. A. Changes in tumor volumes during treatment: Black, vehicle (control mice); Blue; single agent OTX015; Red, single agent targeted drug; Green, OTX015/targeted drug combination. B. Boxplots showing percentage of tumor necrosis at the end of treatment. In each boxplot, the line in the middle of the box represents the median and the box extends from the 25 th to the 75 th percentile (interquartile range). $* \mathrm{P}<0.05$ when compared with control (CTR) mice. C. Histopathological analysis revealed control mice or treated only with rituximab displayed vital cell with a diffuse growth pattern (upper and lower left); addition of OTX015 was associated with large areas of coagulative necrosis (Haematoxyln and Eosin, 200X). 
using a digital caliper. Tumor volumes were calculated as previously described [27]. Mice maintenance and animal experiments were performed with study protocols approved by the local Swiss Cantonal Veterinary Authority (No. 10/2014). Tumor specimens were collected at the end of treatment. Necrosis was semi-quantitatively assessed on hematoxylin-eosin stained slides. The percentage of necrotic cells on the total amount of the neoplastic tissue was evaluated on the whole section in 3 mice per each group. Differences in tumor volumes and percentage of necrosis were calculated using the Wilcoxon rank-sum test (Stata/SE 12.1 for Mac, Stata Corporation).

For OTX015 plasma and tissue concentrations, samples were collected $4 \mathrm{~h}$ after the last OTX015 treatment. Mice were sacrificed and blood was collected from the heart in heparinized tubes, separated immediately by centrifugation $\left(4000 \mathrm{rpm}, 15 \mathrm{~min}, 4^{\circ} \mathrm{C}\right.$ ), and stored at $-80^{\circ} \mathrm{C}$. Plasma concentrations were measured using a validated Ultra Performance Liquid Chromatography with tandem Mass Spectrometry method, as previously described [28]. For tissue measurements, frozen samples were weighed and then homogenized in $1 \mathrm{~mL}$ of water, and

A
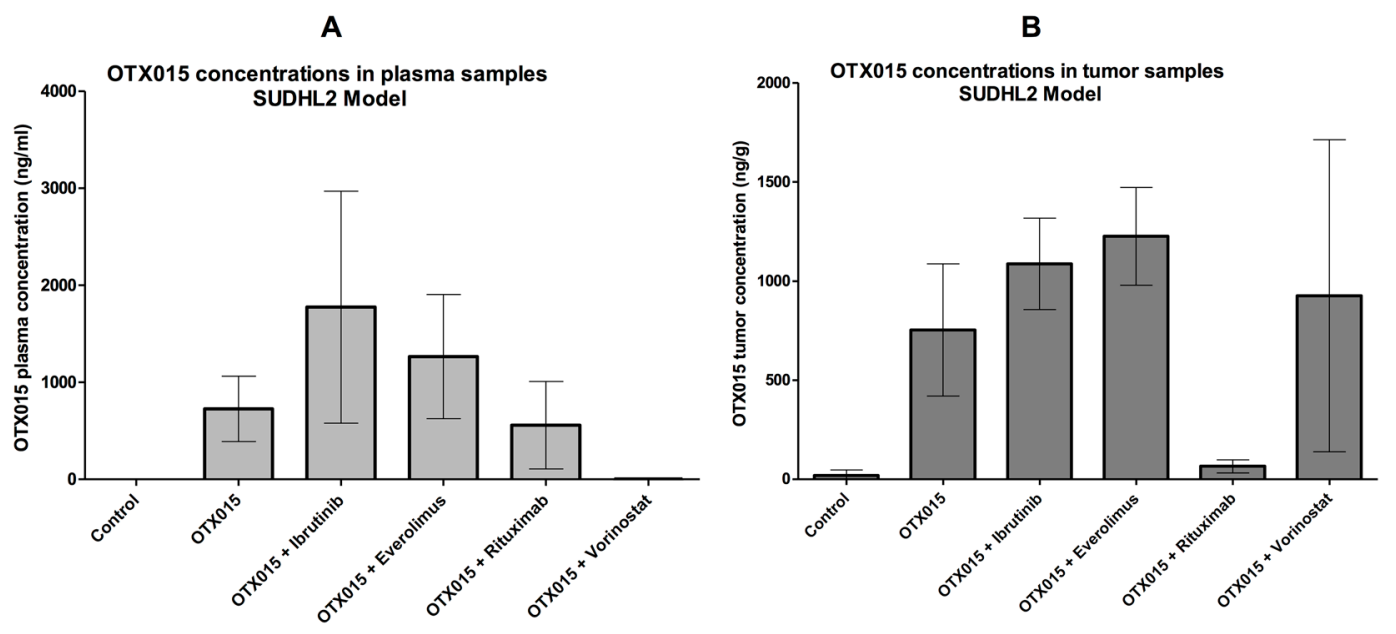

\begin{tabular}{|c|c|c|c|c|c|c|c|c|c|c|c|c|}
\hline SD & 0.93 & 335.7 & 1194.0 & 639.1 & 450.4 & 4.1 & 27.3 & 333.1 & 230.1 & 247.1 & 32.7 & 788.1 \\
\hline $\mathrm{n}$ & 4 & 7 & 6 & 6 & 6 & 6 & 2 & 7 & 6 & 6 & 5 & 6 \\
\hline
\end{tabular}

Figure 2: OTX015 levels in plasma and tumor tissue of SUDHL2-tumor bearing mice as a single agent or in combination with other agents. A. Plasma and B. tissue levels of OTX015 (oral $50 \mathrm{mg} / \mathrm{kg} / \mathrm{day}$ ) were measured after 5 weeks treatment, $4 \mathrm{~h}$ after the last OTX015 administration. Each bar represents the mean \pm standard deviation.

a $50 \mu \mathrm{L}$ sample was prepared using the same extraction method as that used for plasma samples.

\section{ACKNOWLEDGMENTS}

Work partially supported by research funds from Oncoethix, Nelia et Amadeo Barletta Foundation and Gelu Foundation. The authors would like to thanks Sarah MacKenzie (medical writer funded by Oncology Therapeutic Development) and our colleague Afua Adjeiwaa Mensah (Bellinzona, Switzerland) for manuscript editing. This work is lovely dedicated to the memory of Pietro Gaudio, who died on March 11th, 1996.

\section{CONFLICTS OF INTEREST}

FB, KR, AS and EZ have received institutional research funds from Oncology Therapeutic Development. Esteban Cvitkovic was founder and CSO of Oncoethix SA, and CEO of Oncology Therapeutic Development. Maria E. Riveiro was an employee of Oncology Therapeutic Development. EZ has received research funds from Celgene, Novartis, Mundipharma, Roche, Pharmacyclics 
Inc., Johnson \& Johnson's Janssen Pharmaceutical, Gilead. The remaining authors have no conflicts of interest.

\section{REFERENCES}

1. Swerdlow SH, Campo E, Harris NL, Jaffe ES, Pileri A, Stein H, Thiele J, Vardiman JW. (2008). WHO Classification of Tumours of Haematopoietic and Lymphoid Tissues. (Lyon, France: IARC Press), pp. 439.

2. Sehn LH, Gascoyne RD. Diffuse large B-cell lymphoma: optimizing outcome in the context of clinical and biologic heterogeneity. Blood. 2015; 125:22-32.

3. Testoni M, Zucca E, Young KH, Bertoni F. Genetic lesions in diffuse large B-cell lymphomas. Ann Oncol. 2015; 26:1069-1080.

4. Swerdlow SH, Campo E, Pileri SA, Harris NL, Stein H, Siebert R, Advani R, Ghielmini M, Salles GA, Zelenetz AD, Jaffe ES. The 2016 revision of the World Health Organization classification of lymphoid neoplasms. Blood. 2016; 127:2375-2390.

5. Dunleavy K, Roschewski M, Wilson WH. Precision Treatment of Distinct Molecular Subtypes of Diffuse Large B-cell Lymphoma: Ascribing Treatment Based on the Molecular Phenotype. Clin Cancer Res. 2014; 20:5182-5193.

6. Wilson WH, Young RM, Schmitz R, Yang Y, Pittaluga S, Wright G, Lih CJ, Williams PM, Shaffer AL, Gerecitano J, de Vos S, Goy A, Kenkre VP, et al. Targeting B cell receptor signaling with ibrutinib in diffuse large B cell lymphoma. Nat Med. 2015; 21:922-926.

7. Jiang Y, Hatzi K, Shaknovich R. Mechanisms of epigenetic deregulation in lymphoid neoplasms. Blood. 2013; 121:4271-4279.

8. Cerchietti L, Leonard JP. Targeting the epigenome and other new strategies in diffuse large B-cell lymphoma: beyond R-CHOP. Hematology Am Soc Hematol Educ Program. 2013; 2013:591-595.

9. Clozel T, Yang S, Elstrom RL, Tam W, Martin P, Kormaksson M, Banerjee S, Vasanthakumar A, Culjkovic B, Scott DW, Wyman S, Leser M, Shaknovich R, et al. Mechanism-based epigenetic chemosensitization therapy of diffuse large B-cell lymphoma. Cancer Discov. 2013; 3:1002-1019.

10. Lue JK, Amengual JE, O'Connor OA. Epigenetics and Lymphoma: Can We Use Epigenetics to Prime or Reset Chemoresistant Lymphoma Programs? Curr Oncol Rep. 2015; 17:40.

11. Chaidos A, Caputo V, Karadimitris A. Inhibition of bromodomain and extra-terminal proteins (BET) as a potential therapeutic approach in haematological malignancies: emerging preclinical and clinical evidence. Ther Adv Hematol. 2015; 6:128-141.

12. Chapuy B, McKeown MR, Lin CY, Monti S, Roemer MG, Qi J, Rahl PB, Sun HH, Yeda KT, Doench JG, Reichert E,
Kung AL, Rodig SJ, et al. Discovery and characterization of super-enhancer-associated dependencies in diffuse large B cell lymphoma. Cancer Cell. 2013; 24:777-790.

13. Ceribelli M, Kelly PN, Shaffer AL, Wright GW, Xiao W, Yang Y, Mathews Griner LA, Guha R, Shinn P, Keller JM, Liu D, Patel PR, Ferrer M, et al. Blockade of oncogenic IkappaB kinase activity in diffuse large B-cell lymphoma by bromodomain and extraterminal domain protein inhibitors. Proc Natl Acad Sci U S A. 2014; 111:11365-11370.

14. Boi M, Gaudio E, Bonetti P, Kwee I, Bernasconi E, Tarantelli C, Rinaldi A, Testoni M, Cascione L, Ponzoni M, Mensah AA, Stathis A, Stussi G, et al. The BET Bromodomain Inhibitor OTX015 Affects Pathogenetic Pathways in Preclinical B-cell Tumor Models and Synergizes with Targeted Drugs. Clin Cancer Res. 2015; 21:1628-1638.

15. Trabucco SE, Gerstein RM, Evens AM, Bradner JE, Shultz LD, Greiner DL, Zhang H. Inhibition of bromodomain proteins for the treatment of human diffuse large B-cell lymphoma. Clin Cancer Res. 2015; 21:113-122.

16. Emadali A, Rousseaux S, Bruder-Costa J, Rome C, Duley S, Hamaidia S, Betton P, Debernardi A, Leroux D, Bernay B, Kieffer-Jaquinod S, Combes F, Ferri E, et al. Identification of a novel BET bromodomain inhibitor-sensitive, gene regulatory circuit that controls Rituximab response and tumour growth in aggressive lymphoid cancers. EMBO Mol Med. 2013; 5:1180-1195.

17. Sun B, Shah B, Fiskus W, Qi J, Rajapakshe K, Coarfa C, Li L, Devaraj SG, Sharma S, Zhang L, Wang ML, Saenz DT, Krieger S, et al. Synergistic activity of BET protein antagonist-based combinations in mantle cell lymphoma cells sensitive or resistant to ibrutinib. Blood. 2015; 126:1565-1574.

18. Coude MM, Braun T, Berrou J, Dupont M, Bertrand S, Masse A, Raffoux E, Itzykson R, Delord M, Riveiro ME, Herait P, Baruchel A, Dombret H, et al. BET inhibitor OTX015 targets BRD2 and BRD4 and decreases c-MYC in acute leukemia cells. Oncotarget. 2015; 6:17698-17712. doi: 10.18632/oncotarget.4131.

19. Henssen A, Althoff K, Odersky A, Beckers A, Koche R, Speleman F, Schafers S, Bell E, Nortmeyer M, Westermann F, De Preter K, Florin A, Heukamp L, et al. Targeting MYCN-Driven Transcription By BET-Bromodomain Inhibition. Clin Cancer Res. 2016; 22:2470-2481.

20. Sahai V, Redig AJ, Collier KA, Eckerdt FD, Munshi HG. Targeting bet bromodomain proteins in solid tumors. Oncotarget. 2016. doi: 10.18632/oncotarget.9804.

21. Noel JK, Iwata K, Ooike S, Sugahara K, Nakamura H and Daibata M. Development of the BET bromodomain inhibitor OTX015. Mol Cancer Ther. 2013; 12:C244.

22. Loven J, Hoke HA, Lin CY, Lau A, Orlando DA, Vakoc CR, Bradner JE, Lee TI, Young RA. Selective inhibition of tumor oncogenes by disruption of super-enhancers. Cell. 2013; 153:320-334. 
23. Hnisz D, Abraham BJ, Lee TI, Lau A, Saint-Andre V, Sigova AA, Hoke HA, Young RA. Super-enhancers in the control of cell identity and disease. Cell. 2013; 155:934-947.

24. Whyte WA, Orlando DA, Hnisz D, Abraham BJ, Lin CY, Kagey MH, Rahl PB, Lee TI, Young RA. Master transcription factors and mediator establish super-enhancers at key cell identity genes. Cell. 2013; 153:307-319.

25. Amorim S, Stathis A, Gleeson M, Iyengar S, Magarotto V, Leleu X, Morschhauser F, Karlin L, Broussais F, Rezai K, Herait P, Kahatt C, Lokiec F, et al. Bromodomain inhibitor OTX015 in patients with lymphoma or multiple myeloma: a dose-escalation, open-label, pharmacokinetic, phase 1 study. Lancet Haematol. 2016; 3:e196-204.

26. Berthon C, Raffoux E, Thomas X, Vey N, Gomez-Roca C, Yee K, Taussig DC, Rezai K, Roumier C, Herait P,
Kahatt C, Quesnel B, Michallet M, et al. Bromodomain inhibitor OTX015 in patients with acute leukaemia: a dose-escalation, phase 1 study. Lancet Haematol. 2016; 3:e186-195.

27. Gaudio E, Tarantelli C, Kwee I, Barassi C, Bernasconi E, Rinaldi A, Ponzoni M, Cascione L, Targa A, Stathis A, Goodstal S, Zucca E, Bertoni F. Combination of the MEK inhibitor pimasertib with BTK or PI3K-delta inhibitors is active in preclinical models of aggressive lymphomas. Ann Oncol. 2016; 27:1123-1128.

28. Odore E, Lokiec F, Weill S, Noel JK, Herait P, Bekradda M, Riveiro ME, Rezaï K. Development and validation of an UPLC-MS/MS method for quantitative analysis of OTX015 in human plasma samples. Anal Methods. 2014; 6:9108-9115. 